\title{
Producción de Hidrógeno a partir de la fermentación de residuos agroindustriales de la piña
}

\section{Hydrogen production from the fermentation of pineapple agroindustrial wastes}

\section{Luisa Montoya-Pérez ${ }^{1}$, J. Esteban Durán-Herrera²}

Fecha de recepción: 29 de julio de 2016

Fecha de aprobación: 17 de setiembre de 2016

Montoya-Pérez, L; Durán-Herrera, E. Producción de hidrógeno a partir de la fermentación de residuos agroindustriales de la piña. Tecnología en Marcha. Vol. 30-3. Julio-Setiembre 2017. Pág 106-118.

DOI: $10.18845 / \mathrm{tm} . v 30 i 3.3277$ 


\title{
Palabras clave
}

Producción de hidrógeno; fermentación anaeróbica; residuos agroindustriales; corazón de piña; biohidrógeno.

\section{Resumen}

Se estudió la generación de hidrógeno a partir de la fermentación anaeróbica de residuos agroindustriales de corazón de piña utilizando como inóculo lodos pretratados provenientes de una planta de tratamiento de agua residual del tipo UASB. Mediante un diseño factorial $2^{3}$, se determinaron los efectos de la concentración inicial de sustrato, del pH inicial del medio de fermentación y de la formulación de nutrientes, sobre el rendimiento en la producción de hidrógeno. Los mejores resultados se obtuvieron utilizando un $\mathrm{pH}$ inicial de 5,5, con una concentración inicial de sustrato de 5 (g de glucosa)/L y usando una formulación de nutrientes basada en magnesio, hierro, zinc y sodio. Posteriormente, utilizando las condiciones de operación anteriores, se realizó un escalamiento de la fermentación en un bioreactor de $5 \mathrm{~L}$ y se alcanzó un rendimiento máximo en la producción de hidrógeno de 1,541 $\left(\mathrm{mol} \mathrm{H} \mathrm{H}_{2}\right) /(\mathrm{mol}$ glucosa). Este rendimiento es comparable con datos reportados en la literatura cuando se ha usado glucosa como sustrato. Los resultados obtenidos sugieren que los residuos de corazón de piña podrían ser utilizados como una fuente renovable para la producción de hidrógeno.

\section{Keywords}

Hydrogen production; anaerobic fermentation; pineapple wastes; biohydrogen.

\begin{abstract}
Hydrogen production from the fermentation of pineapple core wastes was studied using pretreated sludge from a local UASB wastewater treatment plant as inoculum. Applying a $2^{3}$ factorial design, the effects of initial substrate concentration, initial fermentation medium $\mathrm{pH}$, and type of nutrient formulation on $\mathrm{H}_{2}$ production were studied. The best results were obtained at $\mathrm{pH}$ of 5,5 , with a substrate concentration of 5 (g glucose)/L, and using a nutrient formulation based on magnesium, iron, zinc, and sodium. Then, utilizing the previous operating conditions, the fermentation was scaled up to a $5 \mathrm{~L}$ bioreactor and a maximum yield of $1,541\left(\mathrm{~mol} \mathrm{H}_{2}\right) /(\mathrm{mol}$ glucose) was achieved. This hydrogen yield is comparable with others reported in the literature when glucose was used as substrate. These results suggest that pineapple core wastes could be used as a renewable source for hydrogen production.
\end{abstract}

\section{Introducción}

La búsqueda de fuentes renovables para la producción de combustibles es sin lugar a duda una de las actividades de investigación de mayor vigencia en la actualidad a nivel mundial. Esto es implusado por múltiples factores tales como el calentamiento global del planeta, los problemas diplomáticos por reservas limitadas de hidrocarburos y por la distribución desigual de los recursos no renovables entre los diferentes países. Con estas nuevas fuentes energéticas renovables se pretende detener el uso desmesurado de los combustibles fósiles (gas natural, carbón y petróleo) y disminuir consecuentemente el impacto ambiental generado por su combustión. 
Entre los posibles combustibles renovables se encuentra el hidrógeno, acarreador de energía limpia, ya que su reacción de oxidación con oxígeno produce $120 \mathrm{~kJ} / \mathrm{g}$ de energía liberando únicamente agua en el proceso. A pesar de que no es un elemento que se encuentra de forma libre sobre la tierra, es uno de los elementos más abundantes de ésta ya que conforma numerosos compuestos químicos tales como el agua y la celulosa.

En la actualidad, cerca de un 95\% de la producción mundial de este gas se obtiene a partir de combustibles fósiles mediante procesos donde se genera gas de síntesis; tal es el caso del reformado con vapor de gas natural o naftas ligeras, gasificación de carbón u oxidación de fracciones petrolíferas pesadas. El otro $5 \%$ de hidrógeno se obtiene por electrólisis del agua (4\%) y de gasificación de biomasa (1\%) [1]. Sin embargo, en el afán de suspender el uso de recursos no renovables y de bajar los costos actuales de su producción, se han venido desarrollando nuevas vías de obtención mediante procesos termoquímicos, procesos fotobiológicos, gasificación de biomasa y nuevas tecnologías de electrólisis del agua, entre otros [2].

Una de las alternativas que ha generado altas expectativas es la producción de hidrógeno a partir de rutas de conversión de biomasa (fuente renovable de energía) mediante procesos biológicos como la fermentación anaerobia. Este proceso consiste en la digestión de material orgánico por medio de microorganismos en ausencia de oxígeno. Entre las ventajas que presenta esta tecnología están la capacidad de usar cualquier sustrato rico en carbohidratos, que sus tasas de producción de hidrógeno son más rápidas que las de otros procesos biológicos, costos de operación menores que otros métodos que requieren de energía lumínica y también que las comunidades microbianas utilizadas están disponibles en aguas residuales, compost anaeróbico o lodos profundos [3]. Estudios sobre la producción de hidrógeno a partir de la fermentación anaeróbica utilizando microorganismos de cepas puras, mixtas o simplemente presentes en aguas residuales, han venido obteniendo resultados satisfactorios con las bacterias de la familia conocida como los Clostridium [4]. Según Wang et al. [5], la fermentación anaeróbica estequiometricamente permite convertir 1 mol de glucosa en 2 moles de ácido acético más 4 y 2 moles de hidrógeno y dióxido de carbono, respectivamente.

$$
\mathrm{C}_{6} \mathrm{H}_{12} \mathrm{O}_{6}+2 \mathrm{H}_{2} \mathrm{O} \rightarrow 2 \mathrm{CH}_{3} \mathrm{COOH}+4 \mathrm{H}_{2}+2 \mathrm{CO}_{2}
$$

Con la reacción anterior se tiene una relación significativamente baja de $\mathrm{CO}_{2} / \mathrm{H}_{2}$ con un valor de 0.5. Lo anterior es deseable porque permite una producción más pura de hidrógeno con una menor emisión de $\mathrm{CO}_{2}$, lo cual será beneficioso finalmente en la completa purificación de la corriente del gas de hidrógeno para las aplicaciones posteriores, como por ejemplo con celdas de combustible [5].

Al analizar el contexto nacional, se tiene que en Costa Rica la industrialización de los productos agrícolas genera un 86\% del total de desechos de los sectores industriales. La utilización de estos residuos como una fuente alternativa de materiales de mayor valor agregado, resultaría ventajosa desde el punto de vista económico y ambiental [6]. La piña es la fruta de mayor producción en Costa Rica y se mantiene como el país de mayor exportación de piña fresca a nivel mundial. Para el año 2006 se tenían 22400 ha sembradas con piña, pero para finales del 2010 esta cifra ya llegaba a las 45000 ha cultivadas. Anualmente se producen 2 millones de toneladas de piña y los residuos sólidos de esta fruta correspondientes al corazón, la corona y la cáscara, equivalen a un $45 \%$ del total de cada fruta procesada [7]. Se pretende entonces estudiar la factibilidad técnica de la producción de hidrógeno a partir de este cuantioso desecho agroindustrial. 


\title{
Materiales y métodos
}

\begin{abstract}
Residuo agroindustrial de corazón de piña
El residuo sólido de corazón de piña fue brindado por la empresa Del Monte de la fábrica de congelados ubicada en Heredia, Costa Rica. Los corazones de piña corresponden a la variedad MD-2 conocida como Dorada Extra Dulce. Estos se extraían directamente del proceso y se usaban el mismo día de su recolección. Se licuó $1 \mathrm{~kg}$ de la materia prima durante 3 minutos y se coló para usarse solamente la fase líquida. En la figura 1 se muestran algunos de los corazones de piña usados como sustrato.
\end{abstract}

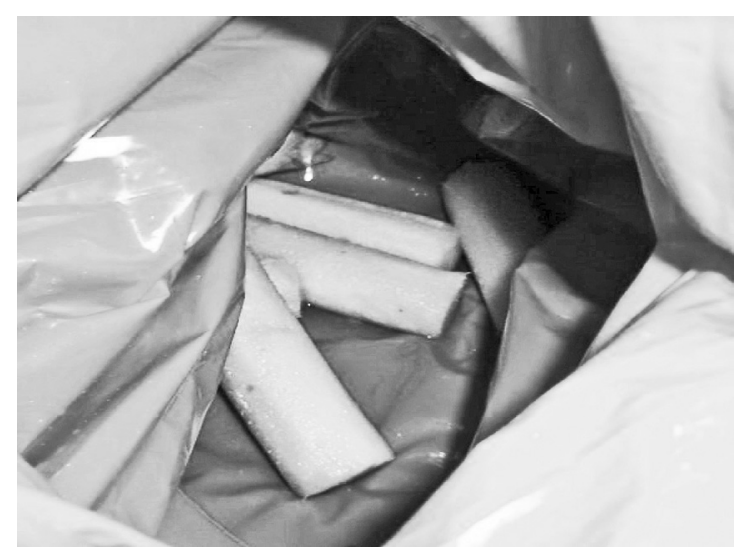

Figura 1. Residuo agroindustrial de corazón de piña

Lodos productores de hidrógeno y formulaciones de nutrientes

Se usaron lodos anaeróbicos extraídos de un reactor UASB de la planta de tratamiento anaerobia de aguas residuales de la empresa Alimentos Prosalud S.A., ubicada en Barranca Puntarenas, Costa Rica. Estos lodos se filtraron haciendo uso de una malla de $2 \mathrm{~mm}$ de apertura y posteriormente fueron tratados térmicamente a $95^{\circ} \mathrm{C}$ por $30 \mathrm{~min}$ antes de su uso [8]. Así mismo se les realizó un análisis microbiológico y una caracterización física según los Métodos Estándar [9].

En la investigación se probaron dos formulaciones de nutrientes que se adicionaron al sustrato de corazón de piña para mejorar los rendimientos [10], [11]; estos se listan en el cuadro 1.

Fermentación anaeróbica en pequeña escala

Se realizó un diseño experimental tipo factorial $2^{3}$ con dos replicas (8 condiciones, 16 experimentos). En los cuadros 2 y 3 se muestran las condiciones experimentales escogidas de las variables de estudio, así como la matriz del diseño. Los experimentos se realizaron en erlenmeyers de $125 \mathrm{~mL}$ tapados con un septum, con un volumen de trabajo de $100 \mathrm{~mL}$. Previo a la inoculación, la mezcla de sustrato y nutrientes se burbujeó con nitrógeno durante 2,5 minutos para obtener una atmósfera anaeróbica en cada erlenmeyer y posteriormente se realizó la esterilización (20 min, $121^{\circ} \mathrm{C}, 101 \mathrm{kPa}$ ). Finalmente las mezclas se inocularon de forma tal de obtener una concentración de lodos al 50\% (v/v), se le ajustó el $\mathrm{pH}$ al valor deseado y se pusieron dentro de una incubadora a una temperatura de $37^{\circ} \mathrm{C}$, sin agitación. El muestro se realizó cada 4 horas en un tiempo de $48 \mathrm{~h}$. 
Cuadro 1. Descripción mineral de las formulaciones de nutrientes Tipo 1 [10] y Tipo 2 [11].

\begin{tabular}{|c|c|c|c|}
\hline \multicolumn{4}{|c|}{ Tipo 1} \\
\hline Componentes & $\begin{array}{c}\text { Cantidad } \\
\text { (mg/L) }\end{array}$ & Componentes & $\begin{array}{c}\text { Cantidad } \\
\text { (mg/L) }\end{array}$ \\
\hline Urea & 4000 & $\mathrm{Na}_{2} \mathrm{MoO}_{4}$ & 10 \\
\hline Peptona & 3000 & $\mathrm{CoCl}_{2} \cdot 6 \mathrm{H}_{2} \mathrm{O}$ & 200 \\
\hline $\mathrm{Na}_{2} \mathrm{HPO}_{4} \cdot 12 \mathrm{H}_{2} \mathrm{O}$ & 21500 & $\mathrm{AlK}\left(\mathrm{SO}_{4}\right)_{2} \cdot 12 \mathrm{H}_{2} \mathrm{O}$ & 10 \\
\hline $\mathrm{KH}_{2} \mathrm{PO}_{4} \cdot 2 \mathrm{H}_{2} \mathrm{O}$ & 8100 & $\mathrm{NiCl} \cdot 6 \mathrm{H}_{2} \mathrm{O}$ & 1 \\
\hline $\mathrm{MgCl}_{2} \cdot 6 \mathrm{H}_{2} \mathrm{O}$ & 100 & $\mathrm{~N}\left[\left(\mathrm{CH}_{2}\right) \mathrm{COOH}\right]_{3}$ & 2000 \\
\hline $\mathrm{FeSO}_{4} \cdot 7 \mathrm{H}_{2} \mathrm{O}$ & 200 & Vitamina B12 & 10 \\
\hline L-cisteína & 100 & Vitamina C & 25 \\
\hline $\mathrm{MnSO}_{4} \cdot 7 \mathrm{H}_{2} \mathrm{O}$ & 10 & Ácido cítrico & 20 \\
\hline $\mathrm{ZnSO}_{4} \cdot 7 \mathrm{H}_{2} \mathrm{O}$ & 50 & Ácido fólico & 10 \\
\hline $\mathrm{H}_{3} \mathrm{BO}_{3}$ & 10 & Creatina & 25 \\
\hline $\mathrm{CaCl}_{2} \cdot 2 \mathrm{H}_{2} \mathrm{O}$ & 10 & Ácido p-aminobenzoico & 10 \\
\hline \multicolumn{4}{|c|}{ Tipo 2} \\
\hline Componentes & $\begin{array}{c}\text { Cantidad } \\
\text { (mg/L) }\end{array}$ & Componentes & $\begin{array}{c}\text { Cantidad } \\
\text { (mg/L) }\end{array}$ \\
\hline Urea & 4000 & $\mathrm{NH}_{4} \mathrm{HCO}_{3}$ & 5240 \\
\hline Peptona & 3000 & $\mathrm{NH}_{4} \mathrm{Cl}$ & 50 \\
\hline $\mathrm{NaCl}$ & 1000 & $\mathrm{NaHCO}_{3}$ & 6720 \\
\hline $\mathrm{MnCl}_{2} \cdot 6 \mathrm{H}_{2} \mathrm{O}$ & 2,5 & $\mathrm{KI}$ & 12,5 \\
\hline $\mathrm{CoCl}_{2} \cdot 6 \mathrm{H}_{2} \mathrm{O}$ & 0,1 & $\mathrm{CuSO}_{4} \cdot 5 \mathrm{H}_{2} \mathrm{O}$ & 5 \\
\hline $\mathrm{Na}_{2} \mathrm{MoO}_{4} \cdot 2 \mathrm{H}_{2} \mathrm{O}$ & 0,1 & $\mathrm{ZnCl}_{2}$ & 0,1 \\
\hline $\mathrm{MnSO}_{4} \cdot 4 \mathrm{H}_{2} \mathrm{O}$ & 2,5 & $\mathrm{CaCl}_{2} \cdot 2 \mathrm{H}_{2} \mathrm{O}$ & 10 \\
\hline $\mathrm{K}_{2} \mathrm{HPO}_{4}$ & 125 & $\mathrm{MgCl}_{2} \cdot 6 \mathrm{H}_{2} \mathrm{O}$ & 120 \\
\hline $\mathrm{FeSO}_{4} \cdot 7 \mathrm{H}_{2} \mathrm{O}$ & 1 & $\mathrm{NiCl}_{2} \cdot 6 \mathrm{H}_{2} \mathrm{O}$ & 2,5 \\
\hline
\end{tabular}

Cuadro 2. Valores de los niveles de las variables de estudio del diseño factorial.

\begin{tabular}{|c|c|c|c|}
\hline Variable & Abreviatura & Nivel Inferior (-) & Nivel superior (+) \\
\hline pH inicial de la mezcla & V1 & 5,5 & 6,5 \\
\hline Concentración inicial de sustrato & V2 & $5 \mathrm{~g} / \mathrm{L}$ & $10 \mathrm{~g} / \mathrm{L}$ \\
\hline Formulación de nutrientes & V3 & Tipo 1 & Tipo 2 \\
\hline
\end{tabular}

\section{Fermentación anaeróbica en reactor de $5 \mathrm{~L}$}

Se realizaron tandas de fermentación en un fermentador de $5 \mathrm{~L}$ de capacidad (Electrolab FerMac 320) un pH inicial de 5,5, con una concentración inicial de sustrato de 5 (g glucosa)/L y usando la formulación de nutrientes Tipo 2. La agitación se fijó en una velocidad de 150 rpm utilizando dos impulsores de turbina de 6 aspas planas y la temperatura se mantuvo en $37^{\circ} \mathrm{C}$ por medio de una chaqueta térmica eléctrica. El volumen de trabajo efectivo fue de $3 \mathrm{~L}$ y la 
preparación del medio fue igual a la descrita anteriormente para las fermentaciones a pequeña escala. El muestreo se realizó a través de un septum ubicado en uno de los puertos localizados en la tapa del fermentador a cada hora posible durante un tiempo de $48 \mathrm{~h}$.

Cuadro 3. Matriz estándar del diseño factorial $2^{3}$ aplicado.

\begin{tabular}{|c|c|c|c|c|c|c|}
\hline \multirow{2}{*}{ Experimento } & \multicolumn{2}{|c|}{ Condiciones V1 } & \multicolumn{2}{c|}{ Condiciones V2 } & \multicolumn{2}{c|}{ Condiciones V3 } \\
\cline { 2 - 7 } & Nivel & Valor & Nivel & Valor & Nivel & Valor \\
\hline 1 & - & 5,5 & - & $5 \mathrm{~g} / \mathrm{L}$ & - & Tipo 1 \\
\hline 2 & + & 6,5 & - & $5 \mathrm{~g} / \mathrm{L}$ & - & Tipo 1 \\
\hline 3 & - & 5,5 & + & $10 \mathrm{~g} / \mathrm{L}$ & - & Tipo 1 \\
\hline 4 & + & 6,5 & + & $10 \mathrm{~g} / \mathrm{L}$ & - & Tipo 1 \\
\hline 5 & - & 5,5 & - & $5 \mathrm{~g} / \mathrm{L}$ & + & Tipo 2 \\
\hline 6 & + & 6,5 & - & $5 \mathrm{~g} / \mathrm{L}$ & + & Tipo 2 \\
\hline 7 & - & 5,5 & + & $10 \mathrm{~g} / \mathrm{L}$ & + & Tipo 2 \\
\hline 8 & + & 6,5 & + & $10 \mathrm{~g} / \mathrm{L}$ & + & Tipo 2 \\
\hline
\end{tabular}

\section{Métodos analíticos}

Para la cuantificación del hidrógeno producido en la fermentación se utilizó un cromatógrafo de gases (Agilent technologies $6890 \mathrm{~N}$ ) equipado con un detector de conductividad térmica y la columna capilar Carboxen-1010 PLOT. La temperatura del puerto de inyección y del detector fueron 200 y $230{ }^{\circ} \mathrm{C}$, respectivamente; la temperatura inicial y máxima de la columna fue 100 y $250{ }^{\circ} \mathrm{C}$, respectivamente, con rampas de temperatura de $15^{\circ} \mathrm{C} / \mathrm{min}$. Se utilizó nitrógeno como gas acarreador a un flujo de $4 \mathrm{~mL} / \mathrm{min}$ y presión de 4,97 psi. Las condiciones de trabajo se basan en una adaptación del método propuesto por Buitrón y Carvajal [12].

Para la caracterización fisicoquímica del corazón de piña se usaron los métodos presentados en el cuadro 4.

Cuadro 4. Métodos empleados para la caracterización fisicoquímica del residuo agroindustrial de corazón de piña.

\begin{tabular}{|c|c|}
\hline Propiedad & Método / Norma \\
\hline Humedad & Horno gravimétrico / JIS 1557-70 \\
\hline Cenizas & Amador [13] / ASTM D1102-56 \\
\hline Fibra cruda & CINA / AOAC 962.09 \\
\hline Azúcares Reductores & Método espectrofotométrico de Nelson Somogyi \\
\hline Glucosa & Método espectrofotométrico de Trinder \\
\hline pH & pH-metro \\
\hline Sólidos Solubles & Refractómetro PAL-1 \\
\hline [N], P, Ca, Mg, K, S, Fe, Cu, Zn, Mn, B & [CIA-SC09-01-01-P06], CIA-SC09-01-01-P10 \\
\hline
\end{tabular}


Modelo cinético para la simulación de la generación de hidrógeno

El modelo cinético utilizado para describir los datos experimentales se basa en la ecuación modificada de Gompertz:

$$
H=P m \cdot \exp \left\{-\exp \left[\frac{R m \times e}{P m}(\lambda-t)+1\right]\right\}
$$

donde $\mathrm{H}$ es la cantidad acumulada de $\mathrm{H}_{2}$ producido (mmol) a un tiempo de incubación $t$ (h), Pm es la cantidad del potencial máximo de $\mathrm{H}_{2}$ producido ( $\mathrm{mmol}$ ), e es el número de Euler 2,71828, $\lambda$ es el tiempo requerido para comenzar la producción de $\mathrm{H}_{2}(\mathrm{~h})$ y $\mathrm{Rm}$ es la tasa de producción máxima de $\mathrm{H}_{2}$ (mmol/h). Las constantes cinéticas ( $\mathrm{Pm}, \mathrm{Rm}$ y $\lambda$ ) fueron obtenidas mediante el ajuste del modelo a los datos experimentales mediante regresión no lineal haciendo uso del software Polymath versión 6.0.

\section{Resultados y discusión}

Análisis de los lodos

Al analizar los lodos en tubos PRAS, los cuales se inocularon con lodos con y sin pretratamiento térmico, se determinó que después de 72 horas a $37^{\circ} \mathrm{C}$, ambos tubos presentaron especies de bacterias mixtas en la tinción de Gram: bacilos Gram positivos esporulados con esporas centrales, subterminales y terminales; bacilos Gram negativos y cocos Gram positivos. También, después de 48 horas a $37^{\circ} \mathrm{C}$ se obtuvo que las placas de agar sangre en atmósfera aerobia presentaron morfología colonial mixta: bacilos Gram negativos entéricos y no entéricos como Bacillus s.p., Streptococcus s.p. y Staphylococcus s.p. En las placas de agar sangre con Neomicina en atmósfera anaerobia, las dos placas presentaron crecimiento de un tipo de Clostridium s.p. casi en cultivo puro e idéntico en ambos casos, formador de swarming. Adicional a los exámenes microbiológicos, se hizo una caracterización física de los lodos madre cuyos resultados aparecen en el cuadro 5.

Cuadro 5. Sólidos sedimentables totales, sólidos suspendidos volátiles, sólidos suspendidos totales y densidad aparente de los lodos madre usados

\begin{tabular}{|c|c|c|c|}
\hline $\begin{array}{c}\text { Sólidos sedimentables } \\
\text { Totales } \\
(\mathrm{mL} \text { lodo/mL muestra) }\end{array}$ & $\begin{array}{c}\text { Sólidos } \\
\text { suspendidos } \\
\text { volátiles }(\mathrm{g} / \mathrm{L})\end{array}$ & $\begin{array}{c}\text { Sólidos suspendidos } \\
\text { totales }(\mathrm{g} / \mathrm{L})\end{array}$ & $\begin{array}{c}\text { Densidad } \\
\text { aparente } \\
(\mathrm{g} / \mathrm{mL})\end{array}$ \\
\hline $7,5 / 35$ & 1,7086 & 2,3257 & 0,0109 \\
\hline
\end{tabular}

Caracterización fisicoquímica del residuo agroindustrial de corazón de piña

En el cuadro 6 se muestra la composición fisicoquímica obtenida en la caracterización del corazón de piña utilizado como materia prima en la investigación.

Se obtuvo que en cada gramo de corazón de piña hay 0,113 g de azúcares reductores y 0,0114 $\mathrm{g}$ de glucosa. Por esta razón es que el residuo agroindustrial de corazón de piña sobresale como un material rico en carbohidratos que se podrían llegar a fermentar fácilmente para la producción de hidrógeno u otros metabolitos de interés. 
Cuadro 6. Composición fisicoquímica del corazón de piña usado como sustrato

\begin{tabular}{|c|c|c|c|}
\hline Propiedad & Cantidad promedio & Desviación estándar & Unidad \\
\hline Humedad & 86,14 & 1,43 & $\%$ \\
\hline Cenizas (base seca) & 1,31 & 0,24 & $\%$ \\
\hline Fibra cruda (g/100 g muestra) & 8,3 & 0 & $\%$ \\
\hline Azúcares Reductores (1 g piña) & 0,113 & 0,002 & $\mathrm{~g} / \mathrm{L}$ \\
\hline Glucosa (40 g piña) & 0,455 & 0,029 & $\mathrm{~g} / \mathrm{L}$ \\
\hline pH & 3,90 & 0 & $\mathrm{pH}$ \\
\hline Sólidos Solubles & 10,03 & 0,81 & $\mathrm{Brix}$ \\
\hline Nitrógeno (base seca) & 0,58 & 0 & $\%$ \\
\hline Fósforo (base seca) & 0,05 & 0 & $\%$ \\
\hline Calcio (base seca) & 0,06 & 0,01 & $\%$ \\
\hline Magnesio (base seca) & 0,12 & 0,01 & $\%$ \\
\hline Potasio (base seca) & 1,24 & 0,35 & $\%$ \\
\hline Azufre (base seca) & 0,07 & 0,01 & $\mathrm{mg} / \mathrm{kg}$ \\
\hline Hierro (base seca) & 23,5 & 10,6 & $\mathrm{mg} / \mathrm{kg}$ \\
\hline Cobre (base seca) & 9,00 & 0 & $\mathrm{mg} / \mathrm{kg}$ \\
\hline Zinc (base seca) & 43,5 & 51,62 & $\mathrm{mg} / \mathrm{kg}$ \\
\hline Manganeso (base seca) & 81,5 & 44,55 & $\mathrm{mg} / \mathrm{kg}$ \\
\hline Boro (base seca) & 5,50 & 0,71 & \\
\hline
\end{tabular}

Fermentaciones en pequeña escala: Efectos de la concentración inicial de sustrato, del pH inicial del medio de fermentación y del tipo formulación de nutrientes sobre el rendimiento en la producción de hidrógeno

Con los datos cinéticos recopilados para cada uno de los 16 experimentos realizados en esta etapa, se procedió a calcular los parámetros cinéticos según la ecuación modificada de Gompertz ( $R m, \lambda$ y $P m$ ), y a partir de ellos el promedio y desviación estándar para cada una de las 8 condiciones (corridas) analizadas. Los valores obtenidos se muestran en el cuadro 7 y a partir de su análisis se observa que bajo las condiciones de las corridas 5, 7 y 8 son con las que hay una mayor producción de hidrógeno (Pm mayores). Sin embargo, hay una diferencia notable en el tiempo de inicio de producción de hidrógeno $(\lambda)$ entre las corridas 5 y $7(\lambda=7,398$ h y 7,906 h), y la 8 ( $\lambda=22,974$ h). Se analiza también que en las corridas $5,6,7$ y 8 llevadas a cabo con el tipo de formulación de nutrientes Tipo 2, se produjo mayor cantidad de hidrógeno que en las corridas 1, 2, 3 y 4 llevadas a cabo con la formulación Tipo 1.

Un aspecto importante de comentar es el hecho que en el análisis de los gases producidos durante el proceso de fermentación, no se detectaron cantidades significativas de metano. Esto indica que el pretratamiento térmico realizado a los lodos, además del pH utilizado en las pruebas, son efectivos para favorecer la producción selectiva de hidrógeno.

Para realizar el análisis estadístico del diseño experimental, se utilizó como variable respuesta el rendimiento en la producción de hidrógeno $\left(\mathrm{Y}_{\mathrm{H} 2}\right)$ con unidades de (mol $\mathrm{H}_{2}$ producido)/(mol de glucosa consumida). Este se calculó dividiendo la constante cinética de máxima producción de hidrógeno $(P m)$ entre los moles consumidos de glucosa en cada corrida. En el cuadro 8 se 
exponen los valores de rendimiento calculados, así como los efectos e interacciones obtenidos en el análisis estadístico. Con los datos de las varianzas entre experimentos se calculó un intervalo de no significancia estadística a un valor de 95\% de confianza, el cual resultó ser $[-0,0064 ; 0,0064]$. Bajo estos resultados, los efectos de las variables 2 (concentración inicial de sustrato) y 3 (formulación de nutrientes) resultaron ser significativos.

Cuadro 7. Parámetros cinéticos estimados para las fermentaciones realizadas

\begin{tabular}{|c|c|c|c|c|c|c|c|c|c|}
\hline \multirow{2}{*}{ Parámetro } & \multicolumn{7}{|c|}{ Condiciones según la matriz estándar del diseño } \\
\cline { 2 - 9 } & 1 & 2 & 3 & 4 & 5 & 6 & 7 & 8 \\
\hline \multicolumn{8}{|c|}{ Promedio } \\
\hline Pm (mmol) & 0,648 & 0,669 & 0,844 & 0,605 & 1,188 & 0,824 & 1,145 & 1,201 \\
\hline Rm (mmol/h) & 4,294 & 0,971 & 0,088 & 3,639 & 0,069 & 0,044 & 0,095 & 0,038 \\
\hline$\lambda(\mathrm{h})$ & 6,972 & 5,601 & 5,889 & 6,317 & 7,398 & 26,123 & 7,906 & 22,97 \\
\hline \multicolumn{8}{|c|}{ Desviación estándar } \\
\hline Pm (mmol) & 0,028 & 0,058 & 0,038 & 0,052 & 0,102 & 0,058 & 0,019 & 0,032 \\
\hline$R m(m m o l / h)$ & 0,038 & 1,279 & 0,002 & 0,114 & 0,000 & 0,004 & 0,020 & 0,003 \\
\hline$\lambda(h)$ & 0,182 & 0,869 & 0,615 & 0,662 & 0,616 & 1,416 & 2,751 & 0,087 \\
\hline
\end{tabular}

Cuadro 8. Rendimiento en la producción de hidrógeno obtenido bajo las diferentes condiciones experimentales, así como sus efectos e interacciones.

\begin{tabular}{|c|c|c|c|c|}
\hline \multirow{2}{*}{ Corrida } & \multicolumn{2}{|c|}{$\mathrm{Y}_{\mathrm{H} 2}$} & \multirow{2}{*}{$\begin{array}{c}\mathrm{Y}_{\mathrm{H} 2} \\
\text { promedio }\end{array}$} & \multirow{2}{*}{$\begin{array}{l}\text { Valor Efecto o } \\
\text { Interacción }\end{array}$} \\
\hline & Peplica I & Replica II & & \\
\hline 1 & 0,0251 & 0,0250 & 0,0250 & $\mu=0,0318$ \\
\hline 2 & 0,0261 & 0,0298 & 0,0280 & $E 1=0,00364$ \\
\hline 3 & 0,0229 & 0,0184 & 0,0206 & $E 2=-0,00861$ \\
\hline 4 & 0,0122 & 0,0328 & 0,0225 & $E 3=0,01548$ \\
\hline 5 & 0,0513 & 0,0455 & 0,0484 & $112=0,00494$ \\
\hline 6 & 0,0443 & 0,0415 & 0,0429 & $113=0,00124$ \\
\hline 7 & 0,0262 & 0,0253 & 0,0257 & $123=-0,00369$ \\
\hline 8 & 0,0418 & 0,0403 & 0,0410 & $1123=0,00549$ \\
\hline
\end{tabular}

En el caso del efecto $2(-0,00861)$ el signo negativo indica que a menores concentraciones de sustrato inicial se favorece el rendimiento en la producción de hidrógeno. Este fenómeno se ha reportado ampliamente en la literatura y se explica debido a procesos de inhibición tanto por el substrato utilizado, como por productos generados durante el proceso de fermentación (ácidos, alcoholes y hasta el mismo hydrógeno) [14]. El valor del efecto $3(0,01548)$ indica que si se hace uso de la formulación de nutrientes Tipo 2 se obtiene mayor rendimiento en la producción de hidrógeno. Además, este efecto presenta el valor absoluto más alto, por lo que se convierte en el efecto más influyente sobre la variable de respuesta. La formulación Tipo 2 está compuesta por elementos metálicos tales como $\mathrm{Zn}, \mathrm{Na}$, Fe y $\mathrm{Mn}$, los cuales ayudan en el transporte y en los cofactores de enzimas, dando mejores resultados en las fermentaciones de producción 
de hidrógeno que en las que se han realizado sobre el medio de cultivo convencional [11]. El efecto 1, asociado al pH inicial de la mezcla, no resultó significativo, por lo que se podría usar en cualquiera de los dos niveles estudiados en una siguiente etapa de experimentación.

Para hacer válida la interpretación de los resultados, los supuestos del modelo estadístico se comprobaron mediante un análisis de residuos. En la figura 2 se muestran las gráficas de calificación normal y de dispersión de los residuos. En la gráfica de calificación normal (figura 2a) los datos se ajustan a una línea recta que pasa por el origen, por esta razón se cumple con el supuesto de normalidad. En la gráfica de distribución normal (figura 2b) se tienen los residuos distribuidos de manera aleatoria sin una tendencia o patrón contundente, por lo que el modelo cumple con el supuesto de varianza constante.
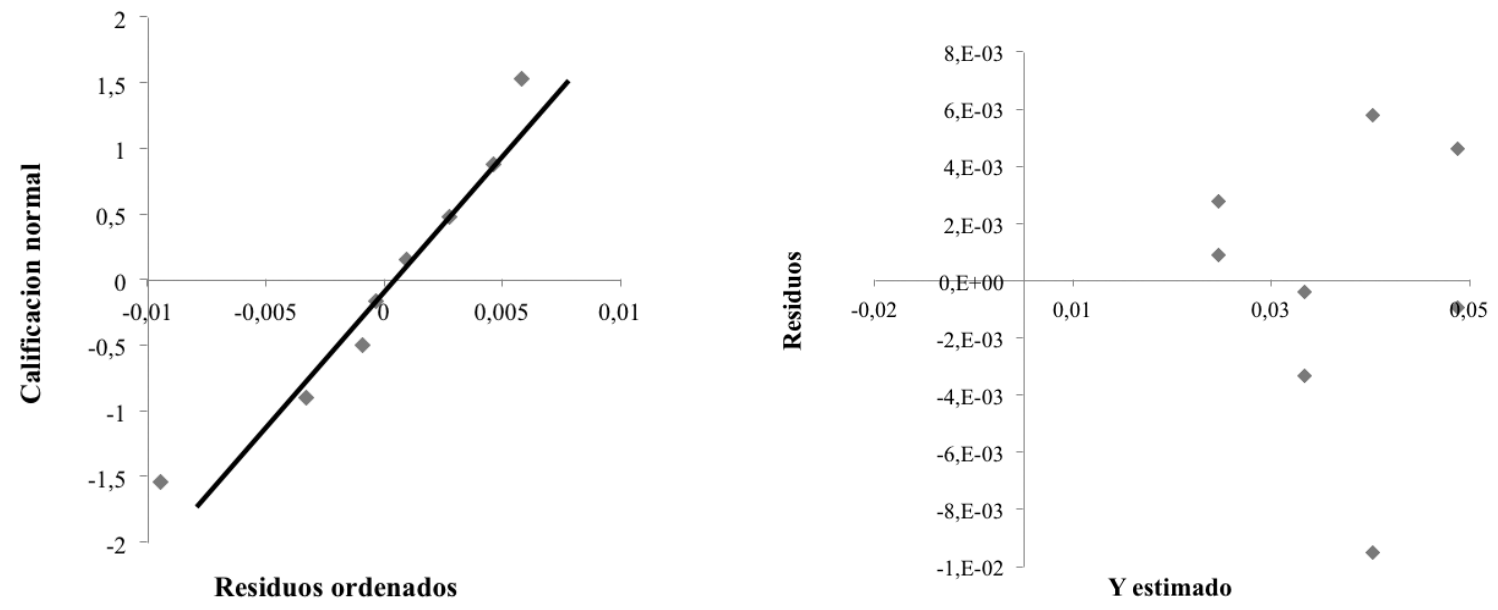

Figura 2. Comprobación de los supuestos del modelo obtenidos para el análisis de residuos: (a) Calificación normal; (b) Varianza constante

Producción de hidrógeno en un fermentador de $5 \mathrm{~L}$

Con la idea de estudiar el proceso de fermentación a una mayor escala, se llevó a cabo el proceso a las condiciones correspondientes a la corrida 5 de la etapa anterior. Esto debido a que bajo estas condiciones se obtuvo el mayor rendimiento $\left(0,0484 \mathrm{~mol} \mathrm{H}_{2} / \mathrm{mol}\right.$ glucosa consumida) y porque cumple con las condiciones deducidas como favorables del análisis estadístico: la variable 2 en su nivel inferior ( $5 \mathrm{~g}$ de glucosa/L) y la variable 3 en su nivel superior (formulación Tipo 2). El pH inicial se estableció en el valor inferior de 5,5. Los datos de producción de hidrógeno obtendos se muestran en la Figura 3, así como la curva de ajuste del modelo cinético utilizado; así mismo, en el cuadro 9, se presentan los valores numéricos obtenidos de las constantes cinéticas $R m, \lambda$ y $P m$.

Como se puede apreciar en la Figura 3 y de los datos del cuadro 9, el tiempo de rezago en la producción de hidrógeno fue de aproximadamente 16 h, la fase exponencial se extiendió hasta aproximadamente las 26 horas y la producción máxima de hidrógeno fue de 41,2 mmol. La fermentación realizada en el reactor de $5 \mathrm{~L}$ se llevó a cabo a un volumen de reacción 30 veces más grande que las realizadas en pequeña escala y hubo una producción de hidrógeno 35 veces mayor. La mayor producción se puede deber a una mejor transferencia de masa en el fermentador de $5 \mathrm{~L}$ debido a la agitación mecánica que se propició, lográndose aprovechar un $8 \%$ más del sustrato inicial. 


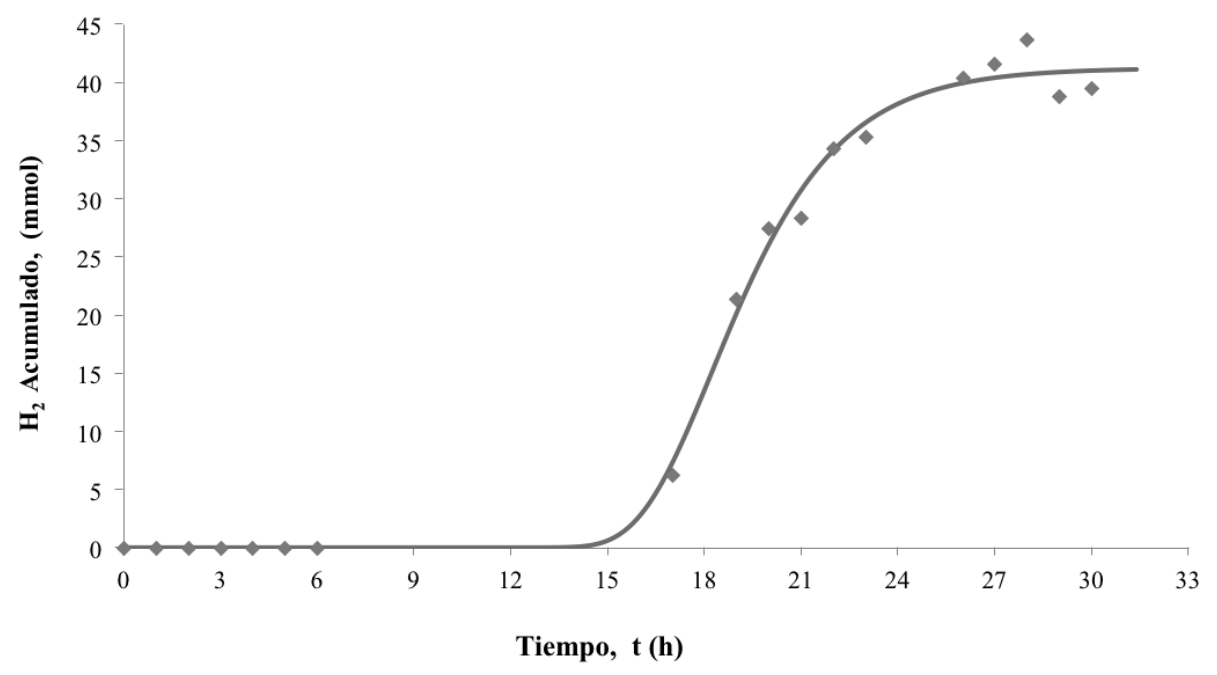

Figura 3. Hidrógeno acumulado obtenido en el fermentador de $5 \mathrm{~L}$

Cuadro 9. Parámetros cinéticos obtenidos para la corrida en el fermentador de $5 \mathrm{~L}$

\begin{tabular}{|c|c|c|}
\hline $\mathrm{Pm}(\mathrm{mmol})$ & $\mathrm{Rm}(\mathrm{mmol} / \mathrm{h})$ & $\lambda(\mathrm{h})$ \\
\hline 41,227 & 6,740 & 16,0 \\
\hline
\end{tabular}

Para las fermentaciones anaeróbicas donde se usan primordialmente bacterias acidogénicas como las Clostridium se tiene un rendimiento teórico de producción de hidrógeno relativamente bajo: $4\left(\mathrm{~mol} \mathrm{H}_{2}\right) /(\mathrm{mol}$ de hexosa), según lo discuten Lo et al. [15]. El rendimiento obtenido en este estudio fue de $1,54\left(\mathrm{~mol} \mathrm{H}_{2}\right) /(\mathrm{mol}$ de glucosa consumida) y representa un $38,5 \%$ del valor teórico que podría llegarse a obtener. Sin embargo, este es un valor que se encuentra dentro del ámbito de rendimientos que se han obtenido en otros estudios reportados en la literatura, tal como se compara en el cuadro 10.

Cuadro 10. Comparación de algunos rendimientos de $\mathrm{H}_{2}$ obtenidos en fermentaciones anaeróbicas en sistemas por tandas reportados en la literatura.

\begin{tabular}{|c|c|c|c|c|}
\hline $\begin{array}{c}\text { Configuración del } \\
\text { sistema }\end{array}$ & Sustrato & Microorganismo & $\begin{array}{c}\mathrm{Y}_{\mathrm{H}_{2}\left(\mathrm{~mol} \mathrm{H}_{2} / \mathrm{mol} \text { de }\right.} \\
\text { S consumido })\end{array}$ & Referencia \\
\hline Batch & Sacarosa & Clostridium butyricum & 2,78 & {$[16]$} \\
\hline Batch & Hexosa añadida & Lodo anaeróbico & 1,29 & {$[17]$} \\
\hline Batch & Glucosa & Clostridium beijerinckii & 1,97 & {$[18]$} \\
\hline Batch & Glucosa & Thermotoga & 0,53 & Citado en [10] \\
\hline Batch & $\begin{array}{c}\text { Extracto desecho } \\
\text { piña }\end{array}$ & Lodo anaeróbico & 1,83 & {$[19]$} \\
\hline Batch & Glucosa & Clostridium butyrium & 2,02 & {$[20]$} \\
\hline Batch & $\begin{array}{c}\text { Extracto desecho } \\
\text { piña }\end{array}$ & Lodo anaeróbico & 1,54 & Este estudio \\
\hline
\end{tabular}


Al caldo final de fermantación se le realizó un análisis de demanda química de oxígeno (DQO) para determinar las características de las eventuales corrientes de desecho de este proceso. El valor obtenido fue de $21105 \mathrm{mg} / \mathrm{L}$, evidenciando el altísimo contenido de materia orgánica aún disponible en ese caldo. Estas mezclas, debido a su rico contenido en ácidos carboxílicos, podrían utilizarse para producir más biohidrógeno a través de procesos fotofermentativos, 0 bien metano, utilizando bacterias metanogénicas.

\section{Conclusiones}

Después de finalizar la presente investigación, se llegó a las siguientes conclusiones:

- El pretratamiento térmico de los lodos y el pH empleado fueron efectivos en impedir el crecimiento de bacterias vegetativas metanogénicas debido a que no se detectó metano significativamente como producto secundario.

- El residuo agroindustrial de corazón de piña sobresale como un material rico en carbohidratos que se puede llegar a fermentar fácilmente para la producción de hidrógeno $\mathrm{u}$ otros metabolitos de interés, conteniendo en promedio 0,113 g de azúcares reductores y 0,0114 g de glucosa por cada gramo de corazón de piña.

- Se obtuvo un rendimiento máximo de producción de hidrógeno de 1,54 $\left(\mathrm{mol} \mathrm{H}_{2}\right) /(\mathrm{mol}$ de glucosa consumida) bajo las siguientes condiciones de trabajo: pH inicial de 5,5, concentración inicial de sustrato de 5 (g glucosa)/L y usando la formulación de nutrientes basada en magnesio, hierro, zinc y sodio.

- El caldo de fermentación final podría reutilizarse para producir más hidrógeno por medio de fotofermentación o para la generación de metano.

- Los resultados obtenidos sugieren que los residuos de corazón de piña podrían ser utilizados como una fuente renovable para la producción de hidrógeno.

\section{Agradecimientos}

Quisiéramos agradecer la valiosa colaboración de las personas que nos ayudaron en los siguientes lugares: Universidad de Costa Rica (Escuela de Ingeniería Química, CELEQ, Laboratorio de Ingeniería Ambiental de la Escuela de Ingeniería Civil, Laboratorio de Tecnología Poscosecha, Laboratorio de Productos Forestales, Facultad de Microbiología y CIPRONA), Congelados Del Monte Costa Rica y Alimentos ProSalud Costa Rica.

\section{Referencias}

[1] H. Balat y E. Kirtay, "Hydrogen from biomass - Present scenario and future prospects", Int. J. Hydrogen Energy, vol. 35, pp. 7416-7426, 2010.

[2] C. M. Kalamaras y A. M. Efstathiou, "Hydrogen Production Technologies: Current State and Future Developments," Conf. Papers in Energy, vol. 2013, Article ID 690627, 9 pages, 2013.

[3] S. Manish y R. Banerjee, "Comparison of biohydrogen production processes", Int. J. Hydrogen Energy, vol. 33, pp. 279- 286, 2008.

[4] G. Davila, S. Arriaga, L. M. Rosales y E. Razo, "Fermentative biohydrogen production: trends and perspectives", Rev. Env. Sci. and Biotech., vol. 7, no. 1, pp. 27-45, 2008.

[5] C. H. Wang, P. J. Lin y J. S. Chang, "Fermentative conversion of sucrose and pineapple waste into hydrogen gas in phosphate-buffered culture seeded with municipal sewage sludge", Process Biochemistry, vol. 41, pp. 1353-1358, 2006. 
[6] M. Moya, "Producción y aprovechamiento de desechos agroindustriales en América Latina" en Development of environmentally compatible polymers from biowaste, Heredia, Costa Rica, 2001, pp. 76-83.

[7] O. Bolaños y H. Hermosilla, "El amargo sabor de la expansión piñera", en Cuaderno de Educación Popular. Limón, Costa Rica: Pastoral Social, Diócesis de Limón, 2005

[8] J. H. Woo y Y. C. Song, "Influence of temperature and duration of heat treatment used for anaerobic seed sludge on biohydrogen fermentation", KSCE J. of Civil Eng., vol. 14, no. 2, pp. 141-147, 2010.

[9] APHA, Standard Methods for the Examination of Water and Wastewater. Washington, DC: American Public Health Association, 1999.

[10] C. M. Pan, Y. T. Fan, P. Zhao y H. W. Hou, "Fermentative hydrogen production by the newly isolated Clostridium Beijerinckii Fanp3", Int. J. Hydrogen Energy, vol. 33, pp. 5383-5391, 2008.

[11] C. Y. Lin y C. H. Lay, "A nutrient formulation for fermentative hydrogen production using anaerobic sewage sludge microflora”, Int. J. Hydrogen Energy, vol. 30, pp. 285-292, 2005.

[12] G. Buitrón y C. Carvajal, "Producción de hidrógeno a partir de aguas residuales", Rev. Digital Univ. UNAM, vol. 10, no. 8, pp. 18-23, 2009.

[13] J. L. Amador, "Evaluación del efecto del tamaño de partícula, tiempo de pretratamiento y concentración de hidróxido de sodio en la hidrólisis enzimática del bagazo de caña de azúcar", Proyecto de graduación, Esc. Ing. Química, Universidad Costa Rica, San José, Costa Rica, 2009.

[14] S. A. Sherif, D. Y. Goswami, E. K. Stefanokos y A. Steinfeld, Handbook of Hydrogen Energy. Boca Raton, Florida, USA: CRC Press, 2014.

[15] Y. C. Lo, C. Y. Chen, C. M. Lee y J. S. Chang, "Sequential dark-photo fermentation and autotrophic microalgal growth for high-yield and $\mathrm{CO}_{2}$-free biohydrogen production", Int. J. Hydrogen Energy, vol. 35, pp. 1094410954, 2010.

[16] W. M. Chen, Z. J. Tseng, K. S. Lee y J. S. Chang, "Fermentative hydrogen production with Clostridium butyricum CGS5 isolated from anaerobic sewage sludge", Int. J. Hydrogen Energy, vol. 30, no. 10, pp. 1063-1070, 2005.

[17] K. W. Jung, D. H. Kim, H. S. Shin, "Continuous fermentative hydrogen production from coffee drink manufacturing wastewater by applying UASB reactor", Int. J. Hydrogen Energy, vol. 35, no. 24, pp. 13370-13378, 2010.

[18] X. Zhao, D. Xing, N. Fu, B. Liu y N. Ren, "Hydrogen production by the newly isolated Clostridium beijerinckii RZF-1108", Bioresour. Technol., vol. 102, no. 18, pp. 8432-8436, 2011.

[19] A. Reungsang y C. Sreela-or, "Bio-Hydrogen Production from Pineapple Waste Extract by Anaerobic Mixed Cultures", Energies, 6, pp. 2175-2190, 2013.

[20] D. Jiang, Z. Fang, S. X. Chin, X. F. Tian y T. C. Su, "Biohydrogen Production from Hydrolysates of Selected Tropical Biomass Wastes with Clostridium Butyricum”, Scientific Reports, vol. 6, Article number: 27205, 2016. 